Pacific Journal of Mathematics

PARTITION AND MODULATED LATTICES 


\section{PARTITION AND MODULATED LATTICES}

\section{DAVID SACHS}

Introduction. The lattice of equivalence relations on a set $S$, or equivalently the lattice of partitions on a set $S$, is perhaps one of the most interesting lattices from the point of view of abstract algebra. Partition lattices were studied rather thoroughly by 0 . Ore [6], who also gave a characterization of them in geometric terms. Later, another characterization of partition lattices was given by U. Sasaki and S. Fujiwara [8]. Their characterization makes specific use of the notions of lines and planes and is somewhat combinatorial in point of view. In this paper we introduce the notion of a modulated lattice and give a characterization of partition lattices (Theorem 14) which is remarkably similar to the lattice-theoretic characterization of the classical projective geometries. Moreover, our study suggests that there may be continuous analogues of partition lattices much in the same way as the continuous geometries of J. Von Neumann are analogues of the classical projective geometries. After developing some preliminary on modulated lattices, we focus our attention on irreducible modulated matroid lattices. A simple property which may or may not be present in such lattices enables us to give our characterization of partition lattices. Curiously enough, we are able to give a characterization of partition lattices on an infinite set which is simpler in appearance than our more general result. We devote some attention to metric lattices and show that certain continuous modulated lattices must be continuous geometries. Finally, we mention some problems and extensions suggested by this paper.

Preliminaries. Let $L$ be a lattice with operations,$+ \cdot$, partially ordered by the relation $\leqq$. The zero (unit) element is written as $0(I)$, and we shall usually assume that these elements are present. We write $(a, b) M$ and say that the pair $(a, b)$ is modular if and only if $(c+a) b=$ $c+a b$ for every $c \leqq b$. If the modular relation is symmetric, then the lattice is said to be semi-modular. If $(a, b) M$ and $a b=0$, then we write $(a, b) \perp$ and say that the pair $(a, b)$ is independent. We say that $b$ covers $c$ if $b>c$ and there is no $x$ for which $b>x>c$. A point is an element which covers 0 . An interval $[a, b]$ is the set of elements $x$ such that $a \leqq x \leqq b$. For the convenience of the reader we include here some properties of semi-modular lattices, the proofs of which can be found in [1] and [3]. All maximal chains between two elements $b, c, b<c$, are finite and have the same length if there exists one finite maximal chain between the two elements [3, p. 88]. By the length of

Received April 25, 1960. This paper was presented to the American Mathematical Society under the title "Modulated and Partition Lattices" on January 29, 1960. 
an interval we mean the number of elements in a finite maximal chain in the interval if there is such a chain. If $(x, y) M$, then the intervals $[x y, x],[y, x+y]$ have the same length if one of the intervals has a finite maximal chain; moreover, if the intervals $[x y, x],[y, x+y]$ have the same length, then $(x, y) M$. An element $c$ is said to have dimension $n$ if the interval [0,c] has length $n+1$; it has codimension $m$ if the interval $[c, I]$ has length $m+1$. By an independent complement of $c$ we mean an element $b$ such that $c+b=I,(c, b) \perp$. By a line we mean an element which covers a point, and by a plane we mean an element which covers a line. A hyperplane is an element which is covered by $I$.

Let $L$ be a lattice with $0 . \quad L$ is left-complemented if for every $a, b \in L$, there exists $b^{\prime} \leqq b$ such that $a+b^{\prime}=a+b,\left(b^{\prime}, a\right) \perp$. A leftcomplemented lattice is semi-modular [10]. Later, we shall show that matroid lattices are left-complemented.

Now let $L$ be a semi-modular lattice. We say that $b$ is a modular element $(b M)$ if $(b, c) M$ for every $c \in L$. The reader can easily verify that $O M, I M$ and $p M$ if $p$ is a point. In the case of affine geometry these are the only modular elements. Evidently a necessary and sufficient condition that every element be modular is that $L$ be a modular lattice.

The meet of two modular elements is modular. For if $a M, b M$, $c \in L$ and $d \leqq a b$, then $(d+c) a b=[(d+c) a] b=(d+c a) b=d+c a b$ since $(c, a) M$ and $(c a, b) M$.

TheOREM 1. Let $a M$. If $b \leqq a$ and $(b, e) M$ relative to $[0, a]$ for every $e \in[0, a]$, then $b M$.

Proof. Notice that $(g, h) M$ in a lattice if and only if $(g, h) M$ relative to the interval $[g h, g+h]$. Let $c \in L, d \leqq b$. Then $(d+c) b=$ $(d+c) b a=[(d+c) a] b=(d+c a) b=d+c a b$ since $a M$ and $c a \leqq a$. But $d+c a b=d+c b$, and this completes the proof.

If $b M$, then the intervals $[b c, b],[c, b+c]$ are isomorphic, and the mappings $x \rightarrow x+c$ and $y \rightarrow y b, x \in[b c, b], y \in[c, b+c]$, are inverse isomorphisms between the two intervals.

\section{Modulated lattices.}

Definition 1. A left-complemented lattice $L$ with unit $I$ is said to be modulated if for every $y M$ and for every $z \geqq y$, there exists $x M$ such that $x+z=I, x z=y$.

Since the zero element is modular, the above conditions cannot be satisfied vacuously. It is easily seen that every complemented modular lattice is a modulated lattice. We shall now give an example of a modulated lattice which is not a complemented modular lattice but is 
describable in terms of such a lattice. Let $A$ be a complemented modular lattice with operations,$+ \cdot$, of length $\geqq 3$ for which every interval sublattice is irreducible and which contains a point $p$. An example of such a lattice is a projective geometry of not necessarily finite length or its dual. We define

$$
L^{\prime}=\Lambda-[p] .
$$

If $L^{\prime}$ is partially ordered in the natural manner, then it is easily seen that $L^{\prime}$ is a lattice. Moreover, if the join and meet operations are denoted by $U$ and $\cap$ respectively, then the following properties hold:

$$
\begin{aligned}
& a \cup b=a+b, \\
& a \cap b=a b \text { if } a b \neq p, \\
& a \cap b=0 \text { if } a b=p .
\end{aligned}
$$

We observe that

(1) $0 \in L^{\prime}, I \in L^{\prime}$;

(2) $x \in L^{\prime}, x \neq 0, y \geqq x$ implies $y \in L^{\prime}$;

(3) $x \in L^{\prime}, y \leqq x$ implies the existence of $z \in L^{\prime}$ such that $z+y=x$, $z y=0$.

We obtain (3) by observing that $[0, x]$ is irreducible, and hence $y$ has at least two complements in $[0, x]$. By a result due to Wilcox [10], $L^{\prime}$ is left-complemented and

$$
(a, b) M \text { if and only if } a b \neq p .
$$

From this we deduce that $a M$ in $L^{\prime}$ if and only if $a=I$ or $a \ngtr p$. Suppose now that $a \neq I$ and $a M$. Let $b>a$. If $b \geqq a+p \equiv c$, then a complement $z$ of $b$ within $[a, I]$ cannot contain $a+p$ so that $z \ngtr p$, and thus $z M$. If $b \ngtr c$, then $b+c$ covers $b$ and therefore $b c=a$. By the irreducibility assumption applied to $[a, b+c]$, there exists $p^{\prime} \neq c$ such that $p^{\prime}+b=b+c, p^{\prime} b=a$. If $x+b+c=I, x(b+c)=p^{\prime}$, then $x b=$ $x(b+c) b=p^{\prime} b=a, x+b=x+p^{\prime}+b=I$. Furthermore, $x c=x(b+c) c=$ $p^{\prime} c \neq c$. Therefore $x \ngtr c$ so that $x \ngtr p$, and $x M$. Since such an $x$ exists, $L^{\prime}$ is a modulated lattice.

THEOREM 2. Let $L$ be a modulated lattice, and let a be a modular element. Then if $c \leqq a,(c, b) M$ relative to $[0, a]$ for all $b \in[0, a]$, and $c \leqq x \leqq a$; there exists an element $y$ such that $y M, x+y=a, x y=c$.

Proof. It follows from Theorem 1 that $c M$. There exists $z \in L$ such that $z+x=I, z x=c, z M$. Define $y \equiv z a$. Then $y M, x+y=$ $x+z a=(x+z) a=a ; x y=x z a=x z=c$.

Since the meet of two modular elements in a semi-modular lattice is a modular element, it follows by induction that the meet of a finite number of modular elements is a modular element. If $L$ has finite 
length, then the modular elements form a lattice; however, this lattice is not usually a sublattice of $L$. It will be shown later that the modular elements of a matroid lattice form a lattice, and our example given above shows there are other examples as well. We shall call the partially ordered system of modular elements $\mathfrak{M}$, and the dual of this system will be denoted by $\overline{\mathfrak{M}}$.

THEOREM 3. If $L$ is a modulated lattice and $\mathfrak{M}$ is a lattice, then $\overline{\mathfrak{M}}$ is a left-complemented lattice.

Proof. If $\mathfrak{M}$ is a lattice, then the meets of elements in $\mathfrak{M}$ and $L$ are the same. The join of two elements $x, y$ in $\mathfrak{M}$ will be denoted by $x \cup y$. Notice that $x \cup y \geqq x+y$ in $L$. Let $a, b \in \mathfrak{M}$. To prove the theorem we need to show the existence of an element $b^{\prime} \geqq b$ such that

$$
\begin{aligned}
a b^{\prime} & =a b, \\
a \cup b^{\prime} & =I
\end{aligned}
$$

and $c\left(b^{\prime} \cup a\right)=c b^{\prime} \cup a$ for every $c \subseteq \mathfrak{M}, c \geqq a$. (This is the dual of leftcomplementation.) Since $L$ is modulated, there exists an element $b^{\prime} M$ such that $(a+b) b^{\prime}=b, a+b+b^{\prime}=I$. Thus $a b=a b^{\prime}, a+b^{\prime}=I$, and so $a \cup b^{\prime}=I$. If $c \geqq a$, then $c\left(b^{\prime} \cup a\right)=c$; also $c\left(b^{\prime}+a\right)=c b^{\prime}+a$, or $c=c b^{\prime}+a$. Thus $c \leqq c b^{\prime} \cup a$, and since the reverse inequality is obvious, we have $c=c b^{\prime} \cup a$. This proves the theorem.

Theorem 4. If $L$ is modulated and $\mathfrak{M}$ is a lattice, then $(a, b) M$ in $\overline{\mathfrak{M}}$ if and only if $a+b=a \cup b$.

Proof. Suppose $a+b=a \cup b$. Then if $c \geqq b, c(a \cup b)=c(a+b)=$ $c a+b$, and so $c(a \cup b) \leqq c a \cup b$. The reverse inequality is obvious, and so $c(a \cup b)=c a \cup b$. Thus $(a, b) M$ in $\overline{\mathfrak{M}}$. Conversely, suppose $a \cup b \neq$ $a+b$. Then $a \cup b>a+b$. If we consider the $b^{\prime}$ in Theorem 3 , then we see that $b^{\prime} a \cup b=b$. But $b^{\prime}(a \cup b)>b$, for if $b^{\prime}(a \cup b)=b$, then $a \cup b=(a \cup b)\left(b^{\prime}+(a+b)\right)=(a \cup b) b^{\prime}+(a+b)=a+b$. Hence $(a, b) M^{\prime}$ in $\overline{\mathfrak{M}}$, and the proof is complete.

If, in our example, $\Lambda$ is the dual of a projective geometry, then $\overline{\mathfrak{M l}}$ is an affine geometry. What we want to show next is that $L$ and $\overline{\mathfrak{M}}$ have the same center if every element in $L$ is a join of points. We first state a few lemmas about left-complemented lattices. The following lemma is easily proved:

LEMMA 1. If $L$ is a left-complemented lattice, $\left(b, b^{\prime}\right) \perp$, and $c=$ $c b+c b^{\prime}$ for every $c \in L$, then $L$ is isomorphic to the cardinal product of $[0, b]$ and $\left[0, b^{\prime}\right]$.

Lemma 2. If $L$ is left-complemented, then an element is in the 
center if and only if it has a unique complement.

Proof. Suppose $e$ has a unique complement $e^{\prime}$. Then $\left(e, e^{\prime}\right) M$. Let $u e=0$. There exists an element $x$ such that $(u+e)+x=I,(u+e, x) \perp$, Now $e+(u+x)=I$ and $e(u+x)=e(u+e)(u+x)=e[(u+e) x+u]=$ $e u=0$. Since $e^{\prime}$ is the unique complement of $e, u+x=e^{\prime}$. Thus if $u e=0$, then $u \leqq e^{\prime}$. For every $x$ there exists an element $b$ such that $e x+e^{\prime} x+b=x,\left(e x+e^{\prime} x, b\right) \perp$. Now $e x\left(e^{\prime} x+b\right)=\left(e x+e^{\prime} x\right)\left(e^{\prime} x+b\right) e x=$ $e^{\prime} x e x=0$. Hence $e\left(e^{\prime} x+b\right)=0$ since $e^{\prime} x+b \leqq x$. It then follows that $e^{\prime} x+b \leqq e^{\prime}$, and so $b \leqq e^{\prime} x$ because $b \leqq x$. Thus $x=e x+e^{\prime} x$ for every $x \in L$. We now use Lemma 1 to see that $e$ is in the center. The converse is trivial.

LEMma 3. Let $L$ be a left-complemented lattice. If e has a unique complement $e^{\prime}$, then e has a unique complement.

Proof. Suppose that $e+e^{\prime}=I,\left(e, e^{\prime}\right) \perp$, and that $e^{\prime}$ is the only element with these properties. If our lemma is false, then there exists an element $b$ such that $e+b=I, e b=0$ and $(e, b) M^{\prime}$. Since there exists $b^{\prime} \leqq b$ such that $e+b^{\prime}=e+b=I,\left(e, b^{\prime}\right) \perp$, it follows that $b>e^{\prime}$. Then there exists $x \leqq b$ such that $e^{\prime}+x=b$ and $\left(e^{\prime}, x\right) \perp$. Also, there exists $x^{\prime} \leqq x$ such that $e+x^{\prime}=e+x$ and $\left(e, x^{\prime}\right) \perp$. Moreover, there exists an element $y$ which is an independent complement of $e+x^{\prime}$. Therefore $e+\left(x^{\prime}+y\right)=I$ and $e\left(x^{\prime}+y\right)=e\left(e+x^{\prime}\right)\left(x^{\prime}+y\right)=e x^{\prime}=0$. If $c \leqq e$, then $\left(c+x^{\prime}+y\right) e=\left[\left(\left(c+x^{\prime}\right)+y\right)\left(e+x^{\prime}\right)\right] e=\left(c+x^{\prime}\right) e=c$. Thus $\left(e, x^{\prime}+y\right) \perp$. From the uniqueness of $e^{\prime}$ we obtain $y+x^{\prime}=e^{\prime}$, and this implies $x^{\prime} \leqq e^{\prime}$. Therefore $x^{\prime} \leqq e^{\prime} x=0$, and then we have $e+x=e$. Thus $x \leqq e b=0, e^{\prime}=b$, and the proof is complete.

THEOREM 5. Let $L$ be a modulated lattice such that every element is a join of points. If $\mathfrak{M}$ is a lattice, then $L$ and $\overline{\mathfrak{M}}$ have the same center.

Proof. If an element $e$ lies in the center of $L$, then it is modular and so it lies in $\overline{\mathfrak{M}}$. Since $e$ has a unique complement $e^{\prime}$ in $L, e^{\prime}$ must be the unique independent complement of $e$ in $\overline{\mathfrak{M}}$; thus $e$ lies in the center of $\overline{\mathfrak{M}}$. Conversely, suppose $e$ lies in the center of $\overline{\mathfrak{M}}$. Then $e$ also lies in the center of $\mathfrak{M}$. Let $e^{\prime}$ be its unique complement in $\mathfrak{M}$. Suppose $c \in L$. Obviously $c \geqq c e+c e^{\prime}$. If $p$ is a point within $c$, then since $p \in \mathfrak{M}, p=p e \cup p e^{\prime}$. Thus $p \leqq e$ or $p \leqq e^{\prime}$. In any case $p=p e+p e^{\prime}$. Hence $p \leqq c e+c e^{\prime}$, and since $c$ is a join of points, $c \leqq c e+c e^{\prime}$. Consequently, $c=c e+c e^{\prime}$ for every $c \in L$. Since $e e^{\prime}=0, e \cup e^{\prime}=I$ and $\left(e, e^{\prime}\right) M$ in $\overline{\mathfrak{M}} ; e+e^{\prime}=I$, and now the result is obvious.

COROLlary. Let $L$ be a modulated lattice such that every element 
is a join of points. If $\mathfrak{M}$ is a lattice, then $L$ is irreducible if and only if $\overline{\mathfrak{M}}$ is irreducible.

\section{Matroid lattices.}

Definition 2. A lattice $L$ is a matroid lattice if it has the following properties:

(4) $L$ is a complete lattice;

(5) Every element in $L$ is the join of points;

(6) If $p$ is a point and $p \$ b$, then $p+b$ covers $b$;

(7) If $p \leqq \sum_{\alpha \in A} p_{\alpha}$ where $p$ and $p_{\alpha}$ are points, then there exists a finite subset $B$ of $A$ such that $p \leqq \sum_{\beta} \in_{B} p_{\beta}{ }^{1}$

A set $Y$ of elements in $L$ is said to be increasingly directed if for every $x, y \in Y$, there exists $z \in Y$ such that $z \geqq x, y$. We define a decreasingly directed set in an analogous manner. If $L$ is a lattice satisfying (4) and (5), then condition (7) is equivalent to the following one:

$$
x \sum_{y \in Y} y=\sum_{y \in Y}(x y)
$$

where $Y$ is an increasingly directed set. We call this property meet continuity. ${ }^{2}$ The proof of the equivalence of (7) and (8) can be found in [3]. We shall now show that a matroid lattice is left-complemented.

Lemma 4. If $p$ is a point, then $(b+p) a=b+p a$ if $b \leqq a$, i.e., $(p, a) M$.

Proof. If $p \leqq b$ or $p \leqq a$, this is obvious. If not, then $b+p$ covers $b$ so that since $b+p \$ a,(b+p) a=b$. But obviously then $(b+p) a=b+p a$.

Lemma 5. Let $a, b \in L$. There exists a maximal element $b^{\prime}$ such that $b^{\prime} \leqq b,\left(b^{\prime}, a\right) \perp$.

Proof. Define

$$
S=[x \in S ; x \leqq b,(x, a) \perp] .
$$

$S$ is partially ordered by the relation $\leqq$ in $L$. If $C$ is a chain in $S$ and $q=\sum_{c \in o} c$, then $q \leqq b$; moreover, a direct application of meet continuity shows that $q a=0$. If $m \leqq a$, then $(m+q) a=\left(m+\sum_{c \in o} c\right) a=$ $\left(\sum_{c \in O}(m+c)\right) a$. Since the set of elements of the form $b+c$ is an

1 A rather through discussion of matroid lattices can be found in [3] where they are called "treillis geometriques". We use the name matroid because it seems to be the more common term.

${ }_{2}^{2}$ This property is sometimes called upper continuity. 
increasingly directed set,

$$
(m+q) a=\sum_{c \in O}(m+c) a=\sum_{c \in O}(m+c a)=m .
$$

Thus $q \in S$, and the existence of $b^{\prime}$ follows from Zorn's lemma.

Lemma 6. If $a, b \in L$, there exists $b^{\prime} \leqq b$ such that $a+b^{\prime}=a+b$, $\left(b^{\prime}, a\right) \perp$.

Proof. There exists a maximal element $b^{\prime} \leqq b$ such that $\left(b^{\prime}, a\right) \perp$. If $a+b^{\prime} \neq a+b$, then there exists a point $p$ such that $p \leqq b, p \geqq a+b^{\prime}$. Since $p$ is a point, $\left(p, b^{\prime}+a\right) \perp$. This implies that $\left(p+b^{\prime}, a\right) \perp$ which contradicts the definition of $b^{\prime}$.

THEOREM 6. A matroid lattice is left-complemented and hence semi-modular.

We state without proof the following structure theorem for matroid lattices:

Structure Theorem. Every matroid lattice is the cardinal product of irreducible matroid lattices; moreover, irreducible matroid lattices are characterized by the fact that any two points have a common complement. ${ }^{3}$

This is the main result of [7], but the theorem was proved in the finite length case in [2] and [4]. Using this theorem, we can easily prove a theorem important for our investigation.

THEOREM 7. If $L$ is an irreducible matroid lattice and $a M$, then $[0, a]$ is an irreducible matroid lattice.

Proof. It is obvious that $[0, a]$ is a matroid lattice. Let $b, c$ be points in $[0, a]$. Since $L$ is irreducible, there exists $x$ such that $b+x=$ $c+x=I, b x=c x=0$. Thus $b+a x=(b+x) a=a=(c+x) a=c+a x$ since $a M$, and $b a x=c a x=0$. Therefore $b$ and $c$ have a common complement in $[0, a]$ from which it follows that $[0, a]$ is irreducible.

CoRollary. If $L$ is an irreducible matroid lattice, $l$ is a line, and $l M$, then $l$ contains at least three distinct points.

THEOREM 8. Let $L$ be a matroid lattice. If $H$ is a set of elements each of which is modular, then $\prod_{h \in H} h \equiv h^{\prime}$ is a modular element.

Proof. The set of all finite meets of elements in $H$ has the same meets as $H$, consists of modular elements, and is decreasingly directed.

${ }^{3}$ By cardinal product we mean cardinal product in the unrestricted sense, i.e., we do not require almost all of the entries to be 0 . 
Thus without loss of generality we assume that $H$ is decreasingly directed, and that its elements are indexed. Let $b$ be an element of finite dimension, and let $c \leqq h^{\prime}$. Thus $c \leqq h_{\beta}$ for every $\beta$. If for every $h_{\gamma}$ there is an $h_{\alpha}$ such that $h_{\alpha}<h_{\gamma}$ and $b h_{\alpha}<b h_{\gamma}$, then there exists an infinite chain between $b$ and 0 . But this is impossible since $b$ has finite dimension. Therefore for some $h_{\gamma}, b h_{\alpha}=b h_{\gamma}$ for every $h_{\alpha}<h_{\gamma}$. Thus $b h^{\prime}=b h_{\gamma}$, and $c+b h^{\prime}=c+b h_{\gamma}=(c+b) h_{\gamma} \geqq(c+b) h^{\prime}$. Hence $h^{\prime}$ is modular with every finite-dimensional element. Let $d^{\prime}$ be any element in $L$. The set $D$ of finite-dimensional elements contained in $d^{\prime}$ is increasingly directed, and its join is $d^{\prime}$. If $c \leqq h^{\prime}$, then we have

$$
\begin{aligned}
\left(c+d^{\prime}\right) h^{\prime} & =\left(c+\sum_{d \in D} d\right) h^{\prime}=\left(\sum_{a \in D}(c+d)\right) h^{\prime}=\sum_{d \in D}(c+d) h^{\prime} \\
& =\sum_{d \in D}\left(c+d h^{\prime}\right)=c+\left(\sum_{a \in D} d h^{\prime}\right)=c+\left(\sum_{a \in D} d\right) h^{\prime}=c+d^{\prime} h^{\prime},
\end{aligned}
$$

where we have used meet continuity twice. This proves the theorem.

Theorem 8 shows that $\overline{\mathfrak{M}}$ is always a lattice, in fact a complete lattice, when $L$ is a matroid lattice. If $L$ is also modulated, then every element in $\overline{\mathfrak{M}}$ is a join of points since $\overline{\mathfrak{M}}$ is left-complemented and every modular element in $L \neq I$ is contained in a modular hyperplane.

THeorem 9. If $X$ is an increasingly directed set of modular elements in a matroid lattice $L$, then $\Sigma X \equiv x^{\prime}$ is a modular element.

Proof. Let $b \in L$, with $c \leqq b$. Then

$$
\begin{aligned}
\left(c+x^{\prime}\right) b & =\left(c+\sum_{x \in X} x\right) b=\left(\sum_{x \in X}(c+x)\right) b=\sum_{x \in X}(c+x) b \\
& =\sum_{x \in X}(c+x b)=c+\left(\sum_{x \in X} x b\right)=c+\left(\sum_{x \in X} x\right) b=c+x^{\prime} b .
\end{aligned}
$$

We shall now restrict our attention to modulated matroid lattices. It is easily shown that a cardinal product of lattices is modulated if and only if each of the factors is modulated. In view of the Structure Theorem we shall therefore concentrate on the irreducible case.

LEMMA 7. Let $L$ be a matroid modulated lattice which is irreducible. Then any two hyperplanes have a common independent complement.

Proof. Let $h^{\prime}, h^{\prime \prime}$ be any two hyperplanes in $L$. We choose a point $p$ such that $p h^{\prime}=0$. If $p h^{\prime \prime}=0$, then $p$ is the common independent complement. If $p \leqq h^{\prime \prime}$, then we choose a line $l M$ such that $l+h^{\prime \prime}=$ $I, l h^{\prime \prime}=p$. Now $l$ must contain at least two more points $r, s$. Neither $r$ nor $s$ can be contained in $h^{\prime \prime}$, for then $l \leqq h^{\prime \prime}$. If both $r$ and $s$ are contained in $h^{\prime}$, then $h^{\prime} \geqq p$ which is false. Hence $r$ or $s$ is a common independent complement. 
THeORem 10. If $L$ is an irreducible modulated matroid lattice, then any two elements of the same dimension (or codimension) have a common independent complement.

Proof. Let $a$ and $b$ have the same dimension or codimension. From the meet continuity condition, there exists a maximal element $x$ such that $(a, x) \perp,(b, x) \perp$. Because $a$ and $b$ have the same dimension or codimension, $a+x=I$ if and only if $b+x=I$. Suppose then that $a+x \neq I, b+x \neq I$. Then $a+x$ and $b+x$ are contained in hyperplanes, and there exists a point $p$ such that $(a+x, p) \perp,(b+x, p) \perp$. One easily sees that $(a, x+p) \perp,(b, x+p) \perp$ contrary to the definition of $x$.

COROLlary. If $L$ is an irreducible modulated matroid lattice, and if $a M, b M$ and $a, b$ have the same dimension or codimension, then $[0, a]$ is isomorphic to $[0, b]$.

Proof. If $x$ is a common independent complement of $a$ and $b$, then $[0, a]$ and $[0, b]$ are both isomorphic to $[x, I]$.

We shall now restrict our attention to irreducible modulated matroid lattices of length $\geqq 5$. Let us consider the following property:

( $\gamma) \quad L$ contains a point $p$ which lies in a plane $t M$ such that three $M$ lines contain $p$ and are contained in $t$.

LEMma 8. Suppose that $L$ is an irreducible matroid modulated lattice of length $\geqq 5$ satisfying condition $(\gamma)$. Let $d M$ be a plane and let $p^{\prime}$ be a point contained in $d$. Then $p^{\prime}$ is contained in three M-lines $l, m, n$ where $l, m, n \leqq d$.

Proof. Suppose that $p^{\prime}=p$ and $d \neq t$. Let $d^{\prime}$ be a common complement of $t$ and $d$. The perspective mapping from $[0, t]$ to $[0, d]$ with $d^{\prime}$ as a common complement leaves $p$ fixed for $\left(p+d^{\prime}\right) d=p+d^{\prime} d=p$ since $p \leqq d$. The images of the three $M$-lines in $t$ containing $p$ are the required lines. (The images are obviously $M$-lines in $[0, d]$ and since $d M$, they are $M$-lines.) Suppose now that $p^{\prime}$ is any point in $t$ distinct from $p$. If $\left(p+p^{\prime}\right) M$, then $p+p^{\prime}$ contains a third point $p^{\prime \prime}$ because $L$ is irreducible. From the length condition, $t$ is contained in a 3 -space $S$ which is modular. From Theorem 2 we see that there exist $M$-planes $t^{\prime}, t^{\prime \prime} \leqq S$ such that $t^{\prime}>p, t^{\prime \prime}>p^{\prime}$ and such that $p^{\prime \prime}$ is a common complement of $t^{\prime}$ and $t^{\prime \prime}$ within [0,S]. Using our previous results, we conclude that $t^{\prime \prime}$ contains three $M$-lines which contain $p^{\prime}$, and a repeated application shows that $t$ contains three $M$-lines which contain $p^{\prime}$. If $p^{\prime}$ is a point for which $\left(p+p^{\prime}\right) M^{\prime}$, then we can find a point $q \leqq t$ such that $(p+q) M$ and $\left(q+p^{\prime}\right) M$. Since $q$ has three $M$-lines in 
$t$ containing it, we conclude that $p^{\prime}$ has the same property. Hence every point in $t$ is contained in three $M$-lines which lie in $t$. We now deduce the conclusion of the theorem by noting that every two $M$-planes are perspectively isomorphic, and that in the isomorphisms $M$-lines map onto $M$-lines.

THEOREM 11. If $L$ is an irreducible matroid modulated lattice of length $\geqq 5$ which satisfies $(\gamma)$, then every line in an M-plane d contains at least three points.

Proof. Let $l$ be such a line. We choose a point $p \leqq d$ such that $p \not l$ and then apply Lemma 8 .

If $L$ is an irreducible modulated matroid lattice of length $\geqq 5$, then we say it satisfies $(\delta)$ if every line in an $M$-plane contains at least three points. Thus Theorem 11 says that $(\gamma)$ implies $(\delta)$.

Lemma 9. Let L satisfy $(\delta)$. If $s M, s$ covers $z, z$ covers $l, z$ covers $m, m M$ and $l \neq m$, then there exists a point $p$ such that $p+l=$ $p+m=z$.

Proof. Since $z$ covers $l, m$ and $(l, m) M ; l, m$ cover $l m$. There exists an element $x M$ such that $x+l m=s, x l m=0$. Clearly the interval $[0, x]$ is isomorphic to the interval $[l m, s]$. Since every line in $[0, x]$ contains three points, there exists an element $r$ such that $l+r=$ $m+r=z, l r=m r=l m$. There exists a point $p$ such that $p+l m=r$. Then $l+p=l+l m+p=l+r=z$, and similarly $m+p=z$.

If $s$ is a modular element, then in view of Theorem $1[0, s]$ is a modular lattice if and only if every element in $[0, s]$ is a modular element.

LEMMA 10. Let $L$ be a matroid lattice, and let $C$ be a chain of elements each of which is modular and has the property that every element contained in it is modular. Then $\Sigma C$ is a modular element, and every element contained in it is modular.

Proof. That $\Sigma C$ is modular follows from Theorem 9. Let $a \leqq \sum C$. The set of elements of the form $a c, c \in C$, is an increasingly directed set of elements with join $\sum_{c \in o} a c=a \sum_{c \in o} c=a$. Each of the elements $a c$ is modular, and therefore $a$ is modular.

We now consider the set of all modular elements $c$ such $[0, c]$ is a modular lattice. According to the preceding lemma and Zorn's lemma, there must exist a maximal such element. The next lemma tells us the character of such maximal elements if $L$ satisfies $(\delta)$. 
Lemma 11. Let $L$ be a lattice satisfying ( $\delta)$. If $l^{\prime}$ is a maximal element with the property that $l^{\prime} M$ and $\left[0, l^{\prime}\right]$ is a modular lattice, then $l^{\prime}$ is a hyperplane or $l^{\prime}=I$.

Proof. Suppose that $l^{\prime}$ is not a hyperplane or $I$. Then there exists an element $Q \neq I$ which is modular and covers $l^{\prime}$. Since $Q \neq I$, there exists a modular element $s$ which covers $Q$. Suppose that $l$ is covered by $Q$, and $l M^{\prime}$. Define $l l^{\prime} \equiv q$. Since $\left(l, l^{\prime}\right) M, l$ covers $q$; moreover, $q$ is modular because $q \leqq l^{\prime}$. There exists $m M$ such that $Q m=q, Q+m=s$, Evidently $m$ covers $q$. Define $z \equiv m+l$; then $z$ covers $l, m$ and is not contained in $Q$. By Lemma 9 there exists a point $p$ such that $p+l=$ $p+m=z$. Since $z \not Q$, it follows that $p \npreceq Q$. There exists an $M$ element $R$ such that $R+z=s, R z=m$. Thus $Q+p=R+p=s$, $Q p=R p=0$. The mapping $x \rightarrow(x+p) Q, x \leqq R$, is an isomorphic mapping from $[0, R]$ onto $[0, Q]$. Evidently $(m+p) Q=(m+l) Q=l+m Q=$ $l+q=l$. Since $m$ is modular with every element in $R, l$ is modular with every element in $Q$. Thus every maximal element in $Q$ is a modular element, and therefore by Theorem 8 every element in $Q$ is a modular element. Thus $[0, Q]$ is a modular lattice with $Q M$, and this contradicts the fact that $l^{\prime}$ was a maximal element with this property.

THEOREM 12. Let $L$ be an irreducible matroid modulated lattice with length $\geqq 5$ satisfying $(\gamma)$ or $(\delta)$. Then if $b \neq I$ and $b M$, then $[0, b]$ is modular lattice.

Proof. If $L$ satisfies $(\gamma)$, then $L$ satisfies $(\delta)$. According to the previous lemma, there exists a hyperplane $h M$ such that $[0, h]$ is a modular lattice. Now if $t M$ and $t$ is a hyperplane, then $[0, t]$ is a modular lattice because $[0, t]$ is isomorphic to $[0, h]$. If $b \neq I$ and $b M$, then $b$ is contained in a modular hyperplane. The result is now evident.

COROLlaRY. If $L$ is an irreducible matroid modulated lattice with length $\geqq 5$, then $L$ satisfies $(\gamma)$ if and only if it satisfies ( $\delta)$. Moreover, $L$ satisfies $(\gamma)$ if and only if $a b \neq 0$ implies $(a, b) M$.

Proof. We have already shown that $(\gamma)$ implies $(\delta)$. Suppose that $L$ satisfies $(\delta)$ and $a b \neq 0$. There exists an element $m M$ such that $a b+m=I, a b m=0$. The interval $[a b, I]$ is isomorphic to $[0, m]$. Since $m \neq I,[0, m]$ is a modular lattice, and therefore $(a, b) M$ within $[a b, I]$. Thus $(a, b) M$.

If $a b \neq 0$ implies $(a, b) M$, then $[p, I]$ is a modular lattice if $p$ is a point. If $h M$ is a complement of $p$, then $[0, h]$ is a modular lattice. Since $h$ must have at least dimension $3,(\gamma)$ follows immediately. 
Partition lattices. The corollary to Theorem 12 tells us a great deal about the irreducible modulated matroid lattices of length $\geqq 5$ which satisfy $(\gamma)$ and also gives us a condition equivalent to $(\gamma)$ which is free of the notion of lines and planes. We digress from our abstract theory to discuss partition lattices.

It is well known [3; p. 265] that partition lattices are matroid lattices. Following Ore [6], we shall call a set of a partition a block and a partition singular if it contains at most one block with more than one element. It is implicit in a result due to Ore [6; p. 583] that the singular partitions are precisely the modular elements. We give here a proof in line with our ideas. If a partition is not singular, then one can easily construct a line which is not modular with it. It is also easily seen that a singular partition which is a hyperplane is a modular element. Since every singular partition is a meet of hyperplane singular partitions, we conclude the result if we use Theorem 8 .

Let $A$ be a singular partition. If $B$ is a partition containing $A$, we can construct a singular partition $B^{\prime}$ as a complement of $B$ within $[A, I]$ as follows:

$$
\begin{aligned}
A & =\left[a_{1}, \cdots, a_{\alpha}\right][a][b][c] \cdots \\
B & =\left[a_{1}, \cdots, a_{\alpha}, \cdots\right]\left[b_{1}, \cdots\right]\left[b_{2}, \cdots\right]\left[b_{\omega}, \cdots\right] \cdots \\
B^{\prime} & =\left[a_{1}, \cdots, a_{\alpha}, b_{1}, b_{2}, b_{\omega}, \cdots\right][e][f] \cdots .
\end{aligned}
$$

That is, we select one element from each of the blocks of $B$ that does not contain the main block of $A$ and combine these elements with the main block of $A$ into one block. If we let $A=0$, i.e., we let $A$ be the partition with all blocks of one element, then we see immediately that $B^{\prime}$ is not unique if $B \neq I, 0$. This shows that a partition lattice is irreducible. Thus partition lattices are irreducible, matroid and modulated.

We now consider the case when $L$ is an irreducible modulated matroid lattice of length $\geqq 5$ and does not satisfy $(\gamma)$. Since $L$ does not satisfy $(\gamma)$, then in a modular plane $P$ some line $l$ contains only two points. The line $l$ is not an $M$-line, for an $M$-line must contain at least three points since $L$ is irreducible. If $t_{1}$ and $t_{3}$ are the points on $l$, then each must be contained in at least two $M$-lines in $P$ because $P$ is an $M$-element. Since $l$ is not an $M$-line, $P$ must contain at least four $M$-lines. If there is a fifth $M$-line, then it cannot meet $l$ at $t_{1}$ or $t_{3}$ because $L$ would then satisfy condition $(\gamma)$. Thus it must meet $l$ at a third point which is impossible. Hence there are exactly four $M$-lines in $P$. If these lines are $l_{1}, l_{2} \geqq t_{1}$ and $l_{3}, l_{4} \geqq t_{3}$, then $t_{1}, t_{3}, l_{1} l_{3}, l_{1} l_{4}, l_{2} l_{3}, l_{2} l_{4}$ are points distinct from each other. There no other points in $P$ because every point must be the meet of two $M$-lines. The plane contains two more lines $l_{1} l_{3}+l_{2} l_{4}, l_{1} l_{4}+l_{2} l_{3}$ and no others because there are no points remaining to make lines. It is easily verified that $P$ is isomorphic to 
the lattice of partitions on a set with four elements. Our next aim is to show that the lattice $\mathfrak{M}$ associated with $L$ is isomorphic to the lattice of singular partitions of some partition lattice.

LEMma 12. If $h \in L$ is an M-element covered by a hyperplane, then $h$ is contained in exactly two M-hyperplanes.

Proof. Since $L$ is modulated, $h$ is contained in at least two $M$ hyperplanes. Suppose it is contained in three $M$-hyperplanes $h_{1}, h_{2}, h_{3}$. Let $p$ be a point such that $p \leqq h$. There exists an $M$-element $m$ such that $m+h=I, m h=p$. Obviously $m$ is a plane. If $m h_{1}=m h_{2}$, then $m h_{1}+h=m h_{2}+h$ or $h_{1}(m+h)=h_{2}(m+h)$ and therefore $h_{1}=h_{2}$. Thus $m h_{1}, m h_{2}, m h_{3}$ are three distinct $M$-lines containing $p$ in $m$. But then $L$ satisfies $(\gamma)$ which is false.

LEMma 13. Every point in $L$ has exactly two M-hyperplanes as complements.

Proof. Since $L$ is irreducible, $\overline{\mathfrak{M}}$ is irreducible, and thus every element in $\overline{\mathfrak{M}}$ must have at least two independent complements. This simply means that every point in $L$ has at least two $M$-hyperplanes as complements. Suppose then that the point $p$ has three $M$-complements $h_{1}, h_{2}, h_{3}$. Now $h_{1} h_{2} \neq h_{2} h_{3}$ because equality would contradict Lemma 12. Thus $h_{1} h_{2}, h_{2} h_{3}, h_{1} h_{3}$ are distinct elements that cover their intersection $h_{1} h_{2} h_{3}$. If $m$ is an $M$-complement of $p+h_{1} h_{2} h_{3}$ within $[p, I]$, then $m \geqq p$, $m+h_{1} h_{2} h_{3}=I$ and $m h_{1} h_{2} h_{3}=0$. Evidently $m$ is a plane, and the intervals $[0, m]$ and $\left[h_{1} h_{2} h_{3}, I\right]$ are isomorphic. Thus $m h_{1}, m h_{2}, m h_{3}$, are three $M$-lines in $m$ which do not contain $p$. But this is impossible because in the lattice of partitions on four elements, any three $M$-lines contain all the points.

LEMMA 14. In a matroid modulated lattice if two elements $b, c$ have the same $M$-complements, then $b=c$.

Proof. Suppose $b \neq c$. Without loss of generality we can assume that $c$ contains a point $p$ which $b$ does not contain. If $m$ is an $M$ complement of $p+b$ within $[p, I]$, then $m \geqq p, m+b=I, m b=0$. But $m c \geqq p$ and the contradiction is apparent.

Lemma 15. Given any two distinct M-hyperplanes $h^{\prime}, h^{\prime \prime}$ in $L$, there exists a point $p$ which is a complement of both $h^{\prime}$ and $h^{\prime \prime}$.

Proof. Let $l$ be an $M$-element which is a complement of $h^{\prime} h^{\prime \prime}$. point on $l$ distinct from $l h^{\prime}$ and $l h^{\prime \prime}$ satisfies the condition. 
Let $G$ be the set of $M$-hyperplanes in $L$.

THEOREM 13. The $\mathfrak{M}$ lattice of $L$ is isomorphic to the lattice of singular partitions of $G$.

Proof. With each point in $L$ we associate the set of elements in $G$ that are complementary to it. The previous lemmas show that each point $p$ is associated with a two element set, different points are associated with different sets, and that every two element subset of $G$ is associated with a point in $L$. Moreover, an $M$-hyperplane contains a point $p$ if and only if it is not a member of the set associated with $p$. Consider the lattice of partitions of the set $G$. If $\alpha$ is an $M$-hyperplane of $L$, then we map it onto the maximal singular partition $[\alpha][\cdots]$. This mapping is obviously one-to-one onto the maximal singular partitions of $G$. If $p$ is a point in $L$ and is associated with the subset $[\beta, \gamma]$ of $G$, then we map it onto the partition $[\beta, \gamma][a][b] \cdots$ where all blocks but the first have one element. This mapping is also one-to-one from the points of $L$ onto the points of the partition lattice of $G$. Now a maximal singular partition $[\alpha][\cdots]$ contains a point partition $[\beta, \gamma][a][b] \cdots$ if and only if $\alpha \notin[\beta, \gamma]$. From this we immediately see that we have defined an order preserving mapping in both directions between the $M$ hyperplanes and points of $L$ and the $M$-hyperplanes and points of the partition lattice on $G$. According to [5; p. 200], two complete lattices in which every element is the join of points and the meet of hyperplanes are isomorphic if the partially ordered sets of hyperplanes and points are isomorphic. It thus follows that the $\mathfrak{M}$ lattice of $L$ is isomorphic to the lattice of singular partitions of $G$.

Definition 3. Let $L^{\prime}$ be a modulated matroid lattice. A set $H$ of elements in $\mathfrak{M l}^{\prime}$ is said to be a quasi-ideal if

(8) $x \in H$ and $y \leqq x$ imply $y \in H$;

(9) $x, y \in H$ and $(x, y) M$ in $\overline{\mathfrak{M}}^{\prime}$ (i.e., $x+y=x \cup y$ ) imply $x \cup y \in H$;

(10) the join of an increasingly directed set of elements in $H$ is also in $H$ (note that join in the sense of $\mathfrak{M l}^{\prime}$ and $L^{\prime}$ are synonymous for increasingly directed sets).

$H$ is a maximal quasi-ideal if in addition it satisfies the following property:

(11) $I \notin H$; if $K$ is a quasi-ideal and $H \subset K$, then $K=H$ or $K=\mathfrak{M}^{\prime}$.

LEMMA 16. If $H$ is the set of elements in $\mathfrak{M}^{\prime}$ contained in a hyperplane $h$ in $L^{\prime}$, then $H$ is a maximal quasi-ideal.

Proof. The first three properties are immediately evident even if $h$ is not a hyperplane. Suppose $H \subset K$ and $H \neq K$. Property (10) 
implies that there exists a maximal element $m$ in $K$ not in $H$ and therefore not contained in $h$. If $m \neq I$, there exists $m^{\prime} M$ such that $m^{\prime}$ covers $m$. Thus $m^{\prime} h+m=m^{\prime}$; consequently there is a point $p \leqq h$ such that $p+m=m^{\prime}$. Thus $m^{\prime} \in K$ by (9) which is impossible.

LEMMA 17. The maximal quasi-ideals of a lattice of singular partitions of a partition lattice $T$ are the set of singular partitions contained in a partition of two blocks.

Proof. It is obvious that the set of singular partitions $\leqq$ a singular partition of two blocks is a maximal quasi-ideal. If we note that two singular partitions form a modular pair in $\overline{\mathfrak{M}}_{T}$ (the $\overline{\mathfrak{M}}$ lattice of $T$ ) if and only if their main blocks overlap, then we readily see that the set of singular partitions $\leqq$ a partition $P$ of two non-trivial blocks is a quasi-ideal. The quasi-ideal determined by $P$ has two maximal singular partitions $m^{\prime}, m^{\prime \prime}$ whose main blocks do not overlap. If a point $p$ is not in $P$, then its main block must overlap the main blocks of $m^{\prime}$ and $m^{\prime \prime}$. Then $\left(p, m^{\prime}\right) M$ in $\overline{\mathfrak{M}}_{T}$, and also $\left(p \cup m^{\prime}, m^{\prime \prime}\right) M$ in $\overline{\mathfrak{M}}_{T}$. Thus $I$ is in any quasi-ideal containing the quasi-ideal determined by $P$ since $p \cup m^{\prime} \cup m^{\prime \prime}=I$, and this proves the maximality of the quasi-ideal determined by $P$. If $Q$ is any quasi-ideal, then in view of (10) it must contain maximal elements. The main blocks of these maximal elements cannot overlap, for otherwise they would be modular in $\overline{\mathfrak{M}}_{T}$ and then they could not be maximal. This observation immediately shows that any quasi-ideal $Q$ must consist of the singular partitions $\leqq$ some partition. But obviously such a quasi-ideal cannot be maximal unless the partition has two blocks.

Lemma 18. Every maximal quasi-ideal in $\mathfrak{M}$ of $L$ is determined by a hyperplane in $L$.

Proof. In view of Lemma 17, every maximal quasi-ideal in $\mathfrak{M}$ of $L$ has one or two maximal elements since $\mathfrak{M}$ is isomorphic to the lattice of the singular partitions of $G$. If a maximal quasi-ideal has one maximal element, then this element must be a hyperplane in $L$ (hyperplanes in $\mathfrak{M}$ are hyperplanes in $L$ ), and the lemma is true in this case. Let $Q$ be a maximal quasi-ideal with two maximal elements $m^{\prime}$ and $m^{\prime \prime}$. In the lattice $\mathfrak{M}, m^{\prime} \cup m^{\prime \prime}=I$. Since $\left(m^{\prime}, m^{\prime \prime}\right) M^{\prime}$ in $\overline{\mathfrak{M}}, m^{\prime} \cup m^{\prime \prime}>m^{\prime}+m^{\prime \prime}$ in $L$. Evidently the set of $M$ elements contained in $m^{\prime}+m^{\prime \prime}$ is a quasiideal containing $Q$, hence equal to $Q$ since $Q$ is maximal. If $m^{\prime}+m^{\prime \prime}$ is not a hyperplane, then there is a hyperplane $h>m^{\prime}+m^{\prime \prime}$. Since $h$ determines a maximal quasi-ideal, $h$ and $m^{\prime}+m^{\prime \prime}$ must determine the same quasi-ideal. But this is impossible, for $h$ contains a point which is not $\leqq m^{\prime}+m^{\prime \prime}$. The proof is complete.

THEOREM 14. A necessary and sufficient condition that a lattice $L$ 
of length $\geqq 5$ be isomorphic to a lattice of partitions of a set $G$ is that

(12) $L$ be an irreducible modulated matroid lattice;

(13) there exist a pair of elements $(a, b)$ such that $(a, b) M^{\prime}, a b \neq 0$.

Proof. Let $L$ satisfy conditions (12) and (13). Our previous results have shown that the $\mathfrak{M}$ lattice of $L$ is isomorphic to the lattice of singular partitions of the set $G$ of modular hyperplanes in $L$. Furthermore, the maximal quasi-ideals of $\mathfrak{M}$ are in a one-to-one correspondence with the hyperplanes of $L$ and the partitions of two blocks in the lattice of partitions of $G$. Thus there is a one-to-one order preserving correspondence in both directions between the hyperplanes and points of $L$ and the hyperplanes and points the lattice of partitions of $G$. From this we conclude that the two lattices are isomorphic.

Conversely, if $L$ is a partition lattice, then it evidently satisfies (12). It is easily shown that a partition lattice of length $\geqq 5$ has two hyperplanes which do not form a modular pair and do not meet in 0 since every interval $[\alpha, I]$ of a partition lattice is itself a partition lattice. The proof is complete.

REMARK. It is impossible for (13) to be satisfied in a matroid lattice of length $\leqq 4$. Hence our condition in Theorem 14 is neither necessary nor sufficient if $L$ has length $\leqq 4$ although (12) is necessary.

A lattice is said to be simple if it has only trivial congruence relations. Obviously a simple lattice is irreducible although the converse is not necessarily true. Ore [6] has shown that a partition lattice is simple. Thus Theorem 14 is still correct if we replace the word "irreducible" by the word "simple". What we intend to show is that if $L$ is of infinite length, then condition (13) may be deleted if simplicity replaces irreducibility in (12).

By a neutral ideal of a relatively complemented lattice $L$ with 0 , we mean an ideal which is the kernel of a homomorphic mapping. Ore [6] has given the following intrinsic characterization of a neutral ideal: an ideal $N$ is neutral if and only if for every $x, y \in L, a \in N$, there exists $b \in N$ such that $x y+b=(x+a) y+b$.

Lemma 19. Let $L$ be a semi-modular lattice. If $b$ covers $a$ and $(a, y) M$, then by $=$ ay or by covers ay.

Proof. If $(a, y) M$, then $(c+a) y b=(c+a y) b=c+a y=c+a y b$ if $c \leqq y b$. Thus $(a, b y) M$. Since $b$ covers $a, a=a+b y$ or $a+b y$ covers $a$. If $a=a+b y$, then $a y \geqq b y$ and therefore $a y=b y$ since $b$ covers $a$. If $a+b y$ covers $a$, then $b y$ covers $a b y=a y$ since if $(u, v) M$ and $u+v$ covers $v$, then $u$ covers $u v$. 
LEMMA 20. Let $L$ be an irreducible modulated lattice of infinite length satisfying $(\gamma)$. Then the set $F$ of all finite-dimensional elements is a neutral ideal.

Proof. It is obvious that $F$ is an ideal. Suppose that $x, y \in L$ and $a \in F$. Let $h$ be an $M$-hyperplane. Since $a$ is finite-dimensional, there exists a finite maximal chain between $x$ and $x+a$. Using the results of Lemma 19 and the fact that $y h$ is an $M$-element (cf. Theorem 12), we see that there is a maximal chain between $x y h$ and $(x+a) y h$ of length no greater than the maximal chain between $x$ and $x+a$. Since $(x+a) y h=(x+a) y$ or $(x+a) y h$ is covered by $(x+a) y$, there exists a finite maximal chain between $x y$ and $(x+a) y$. Let $b$ be an independent complement of $x y$ within $[0,(x+a) y]$. Then $b \in F$ and $(x+a) y+b=$ $x y+b$. This completes the proof.

THEOREM 15. A lattice $L$ of infinite length is isomorphic to a partition lattice if and only if it is a simple modulated matroid lattice.

Proof. The necessity is evident. If $L$ satisfies $(\gamma)$ and is irreducible, then it cannot be simple in view of Lemma 20 .

REMARK. Every projective geometry of finite length is a simple modulated matroid lattice, so that our condition in Theorem 15 is not sufficient if $L$ has finite length.

Metric lattices. By a valuation on a lattice $L$ we mean a realvalued function | | defined for each element in $L$ such that

(14) $x<y$ implies $|x|<|y|$;

(15) $|x+y|+|x y| \leqq|x|+|y|$;

(16) $\quad(x, y) M$ if and only if $|x+y|+|x y|=|x|+|y|$.

As is well known, every semi-modular lattice of finite length has such a valuation. If we define $d(x, y)=2|x+y|-|x|-|y|$, then $L$ becomes a metric space [9] in which $|(|a|-|b|)| \leqq d(a, b)$. Moreover, $d(a+e, b+f) \leqq d(a, b)+d(e, f)$ [9], so that the join operation is a uniformly continuous function. We shall refer to $L$ as a metric lattice and say that $L$ is metrically complete if it is complete in the metric defined above. A metrically complete lattice $L$ is complete as a lattice if it contains 0 and $I$ [9].

Lemma 21. If $(a, x) M$ and $(a, y) M$, then $d(a x, a y) \leqq d(x, y)$. 


$$
\begin{aligned}
\mid a(x+y) & |-| a y \mid \\
& \leqq|a|+|x+y|-|a+x+y|-|a|-|y|+|a+y| \\
& \leqq|x+y|-|y| .
\end{aligned}
$$

Similarly, $|a(x+y)|-|a x| \leqq|x+y|-|x|$. Thus $2|a(x+y)|-|a x|-$ $|a y| \leqq 2|x+y|-|x|-|y|$. But since $a(x+y) \geqq a x+a y,|a(x+y)| \geqq$ $|a x+a y|$. Hence $2|a x+a y|-|a y|-|a x| \leqq 2|x+y|-|x|-|y|$, and this proves the lemma.

THEOREM 16. Let $L$ be a metrically complete lattice. If the sequence $\left(a_{i}\right)$ has limit $a$ and $\left(a_{i}, x\right) M$ for every $i$, then $(a, x) M$ and $\lim$ $\left(a_{i} x\right)=a x$.

Proof. For each $i$ we have $\left|a_{i}+x\right|+\left|a_{i} x\right|=\left|a_{i}\right|+|x|$. Thus. $\left|a_{i} x\right|=\left|a_{i}\right|+|x|-\left|a_{i}+x\right|$. If $\lim \left(y_{i}\right)=y$, then $\lim \left(\left|y_{i}\right|\right)=|y|$, for $\left|\left(|y|-\left|y_{i}\right|\right)\right| \leqq d\left(y, y_{i}\right)$. Since $\lim \left(a_{i}+x\right)=a+x, \lim \left(\left|a_{i}+x\right|\right)=|a+x|$. Therefore $\lim \left(\left|a_{i} x\right|\right)=|a|+|x|-|a+x|$. The sequence $\left(a_{i} x\right)$ is a Cauchy sequence, for $d\left(a_{i} x, a_{j} x\right) \leqq d\left(a_{i}, a_{j}\right)$ since $\left(a_{i}, x\right) M,\left(a_{j}, x\right) M$. Since $L$ is metrically complete, there exists $a^{\prime}$ such that $\lim \left(a_{i} x\right)=a^{\prime}$, and therefore $\lim \left(\left|a_{i} x\right|\right)=\left|a^{\prime}\right|$. Now $\lim \left(a_{i}+a_{i} x\right)=\lim \left(a_{i}\right)=a$. But since the join operation is continuous, we have $\lim \left(a_{i}+a_{i} x\right)=\lim \left(a_{i}\right)+\lim \left(a_{i} x\right)=$ $a+a^{\prime}$. Therefore $a^{\prime} \leqq a$. We also have that $x=\lim \left(x+a_{i} x\right)=$ $\lim (x)+\lim \left(a_{i} x\right)=x+a^{\prime}$. Thus $a^{\prime} \leqq x$ and consequently $a^{\prime} \leqq a x$. Since $|a x| \leqq|x|+|a|-|a+x|$ and $\left|a^{\prime}\right|=|x|+|a|-|a+x|$, it follows that $|a x| \leqq\left|a^{\prime}\right|$. This implies that $a^{\prime}=a x$ and the result follows.

It is to be noted that our proof requires the metric completeness of $L$, and the theorem is false if one does not assume metric completeness. as can be shown in an example in [9]. The reason this is so is that when one metrically completes a lattice, the join operation is preserved. but the meet operation need not be.

We say that a lattice with more than one element is dense if $x>y$ implies the existence of an element $z$ such that $x>z>y$. A left-complemented lattice of length $>1$ is dense if and only if it contains no points. It is easily shown that a maximal chain $C$ in $[a, b]$ of a metrically complete dense lattice is isomorphic and isometric to a closed interval of real numbers.

Let $L$ be a left-complemented lattice. If $a, b$ are contained in $[c, d]$, and have a common independent complement relative to $[c, d]$, then they have a common independent complement relative to any interval containing $[c, d]$.

LEMMA 22. Let $L$ be a left-complemented lattice such that every interval sublattice is irreducible. If $a$ and $b$ are incomparable, then there exists an element $p>0$ such that $(a, p) \perp,(b, p) \perp$. 
Proof. There exist $a^{\prime}, b^{\prime}$ such that $a^{\prime}+b=a+b,\left(a^{\prime}, b\right) \perp, a^{\prime} \leqq a$, $b^{\prime}+a=b+a,\left(b^{\prime}, a\right) \perp, b^{\prime} \leqq b$. Therefore $a^{\prime} \neq 0, b^{\prime} \neq 0$ and $\left(a^{\prime}, b^{\prime}\right) \perp$. Since the interval $\left[0, a^{\prime}+b^{\prime}\right]$ is irreducible and contains more than two elements, there exists $x<a^{\prime}+b^{\prime}$ such that $x\left(a^{\prime}+b^{\prime}\right) \neq x a^{\prime}+x b^{\prime}$. Therefore $x>x a^{\prime}+x b^{\prime}$. Now there exists $u \neq 0$ such that $x a^{\prime}+x b^{\prime}+u=$ $x,\left(x a^{\prime}+x b^{\prime}, u\right) \perp$. Since $u \leqq x, u a^{\prime} \leqq x a^{\prime}$; therefore $u a^{\prime}=u a^{\prime} x a^{\prime}=u x a^{\prime} \leqq$ $u\left(x a^{\prime}+x b^{\prime}\right)=0$. Therefore $u a^{\prime}=0$ and also $u b^{\prime}=0$. But $u a=$ $u\left(a^{\prime}+b^{\prime}\right) a=u a^{\prime}=0$, and in a similar way $u b=0$. We choose $u^{\prime}$ such that $u^{\prime}+a=u+a,\left(u^{\prime}, a\right) \perp, u^{\prime} \leqq u$. Consequently $u^{\prime} \neq 0$. There exists $p$ such that $p+b=u^{\prime}+b,(p, b) \perp, p \leqq u^{\prime}$. Quite obviously $p \neq 0$ and $(p, a) . \perp$. This completes the proof.

THEOREM 17. Let $L$ be a left-complemented metrically complete lattice in which every interval sublattice is irreducible. If $|a|=|b|$, there exists $c \in L$ such that $a+b=a+c=b+c, a c=b c=a b,(a, c) M$, $(b, c) M$.

Proof. Without loss of generality we assume that $a b=0$. The case $a=b$ is trivial. Suppose $a \neq b$. Since $a$ and $b$ are incomparable, there exists $p>0, p \leqq a+b$, such that $(a, p) \perp,(b, p) \perp$. Let $c$ be a maximal element with this property. ${ }^{4}$ If $a+c=a+b$, then $b+c=$ $a+b$; for $|a|=|b|,|a+c|=|c|+|a|-|0|,|b+c|=|c|+|b|-|0|$, and therefore $|a+c|=|b+c|$. Suppose then that $a+b \neq a+c$, $a+b \neq b+c$. If $a+c=b+c$, then $a+c=a+b$. Thus $a+c$ and $b+c$ are incomparable, and therefore there exists $m>0, m \leqq a+b$, such that $(a+c, m) \perp,(b+c, m) \perp$. It is easily seen that $(a, c+m) \perp$, $(b, c+m) \perp$, and this contradicts the definition of $c$.

Let $M$ be an irreducible continuous geometry valuated in such a way that $|I|=2,|0|=0$. If we define $L$ to be the set of all elements $x$ with $|x|<1$ plus $I$ and valuate $L$ so that $|I|=1$ and all other elements. have the same valuation as they do in $M$, then $L$ is a lattice without points which satisfies the hypothesis of Theorem 17 and is not a continuous geometry.

We have already studied metric lattices which are modulated because every semi-modular lattice of finite length has a valuation. To facilitate our study of the irreducible modulated lattices of finite length, we introduced the condition $(\gamma)$ which makes no sense if our lattice has no points. By the use of Theorem 12 one can show that all the intervals except possibly the intervals $[0, l]$ where $l$ is a line are irreducible. We have shown that irreducible lattices which satisfy $(\gamma)$ have the additional property that $a b \neq 0$ implies $(a, b) M$. It is not too difficult to show

${ }^{4}$ Let $S$ be a set of elements in $L$ where $L$ is a metrically complete lattice with 0 and I. Theorem 16 and the fact that every increasing transfinite sequence is countable imply that there exists a maximal element independent of each element of $S$. 
that metrically complete lattices which are dense and have this latter property must be modular lattices if we use Theorem 16. This leads one to suspect that modulated lattices which are dense, metrically complete, and which have no reducible interval sublattices are actually continuous geometries. This is the case as is seen below.

THEOREM 18. Let $L$ be a modulated lattice without points which is metrically complete and contains at least two elements. If every interval sublattice of $L$ is irreducible, then $L$ is an irreducible continuous geometry.

Proof. We assume without loss of generality that our valuation is normalized, i.e., $|0|=0,|I|=1$. Since $L$ is dense and metrically complete, there are elements of any valuation between 0 and 1 ; thus there are modular elements of any value between 0 and 1 . We define a set $S$ of real numbers as follows:

$S=[a \in S ; a \in[0,1] ;$ for every $y M$ with $|y| \leqq a$, the interval $[0, y]$ is a modular lattice].

$S$ is non-empty because $0 \in S$. Let $\omega$ be the least upper bound of $S$. Then $\omega \in S$. To prove this it suffices to consider those modular elements $y$ for which $|y|=\omega$. There exists an increasing sequence $\left(y_{i}\right)$ with limit $y$ such that $y_{i} M$ for each $i$. Let $c \in[0, y]$. Since $c y_{i} M$ and $\left(c, y_{i}\right) M$ for every $i, \lim \left(c y_{i}\right)=c y=c$ and $c M$. Thus $[0, y]$ is a modular lattice. Suppose then that $1-\omega \neq 0$. There exists $\delta$ with $1>\delta>\omega$ such that $1-\delta \geqq \delta-\omega$. To prove the theorem it suffices to show that $\delta \in S$. Let $a$ be an $M$-element with $\omega<|a| \leqq \delta$. Let $c \leqq a$. There exists $q \leqq a$ such that $|q|=\omega$ and $q M$. Evidently $q c M$ since $[0, q]$ is a modular lattice. Since $L$ is a modulated lattice, there exists $d^{\prime}$ such that $a+d^{\prime}=I, a d^{\prime}=q c, d^{\prime} M$. There exists a real number $\alpha$ such that $\alpha-|\alpha|=|c|-|q c|$. Then $\alpha=|a|+|c+q|-|q|$. Thus $\alpha \leqq \delta+$ $\delta-\omega \leqq 1$. But there exists $r \geqq a$ with $r M$ such that $|r|=\alpha$. Since $|r|-|a|=|c|-|q c|$ and $|r|+\left|d^{\prime}\right|=1+\left|r d^{\prime}\right|$, it follows that $\left|d^{\prime}\right|+|a|=1-|c|+\left|r d^{\prime}\right|+|q c|$. Using the fact that $\left|d^{\prime}\right|+|a|=$ $1+|q c|$, we find that $|c|=\left|r d^{\prime}\right|$. If we define $r d^{\prime} \equiv d$, then $a+d=r$, $a d=q c=c d$, and $d M$ since $r M$ and $d^{\prime} M$. From the irreducibility of the interval $[c d, c+d]$ there exists $z \in L$ such that $d+z=c+z=$ $c+d, d z=c z=c d,(c, z) M$ and $(d, z) M$. Now $a z=a(c+d) z=c z=c d$. Also $a+z=a+c+z=a+c+d=a+d=r$. Since $r M$ and $d M$, there exists $b \in L$ such that $c+d+b=r,(c+d) b=d, b M$. Therefore $b z=b(c+d) z=d z=c d, b+z=b+z+d=b+c+d=r$. This shows that $|b|=|a|$. There exists $x \in L$ such that $x+c d=z$ and $(x, c d) \perp$. Then $a+x=a+c d+x=r=b+c d+x=b+x, a x=a z x=c d x=$ $0=b z x=b x$. The mapping $y \rightarrow(y+x) a, y \in[0, b]$, is an isomorphism from $[0, b]$ onto $[0, a]$. Now $d \rightarrow(d+x) a=(d+c d+x) a=(d+z) a=$ 
$(c+z) a=c$. Since $c$ is the image of $d$ under the isomorphism, $c$ is modular with every element in $[0, a]$ and so $c M$. Thus $[0, a]$ is a modular lattice for every $a M$ with $\omega<|a| \leqq \delta$. Hence $\delta$ is in $S$ which is a contradiction. Thus $\omega=1$, and the proof is complete.

Conclusion. It might be of some interest to determine the existence or non-existence of an irreducible metrically complete modulated lattice which is dense but is not a continuous geometry. Such a lattice would be a natural generalization of finite partition lattices in view of our Theorems 14 and 17. In a subsequent paper we shall show how to represent lattices satisfying $(\gamma)$ in terms of projective geometries. Using this representation, we can show that every interval sublattice of a matroid modulated lattice is a modulated lattice.

\section{BIBLIOGRAPHY}

1. Garrett Birkhoff, Lattice Theory, Rev. Ed. New York: Amer. Math. Soc., 1948.

2. R.P. Dilworth, The structure of relatively complemented lattices, Ann. of Math., 51 (1950), 348-59.

3. M. L. Dubreil-Jacotin, L. Lesieur and R. Croisot, Leçons sur la Théorie Des Treillis Des Structures Algebriques Ordonnées et Des Treillis Géométriques. Paris: Gauthier-Villars, 1953.

4. J.E. McLaughlin, Projectivities in relatively complemented lattices, Duke Math. J., 18 (1951), 73-84.

5. - Structure theorems for relatively complemented lattices, Pacific J. Math., 3 (1953), 197-208.

6. O. Ore, Theory of equivalence relations, Duke Math. J., 9 (1942), 573-627.

7. U. Sasaki and S. Fujiwara, The decomposition of matroid lattices, J. Sci. Hiroshima Univ. Ser. A., 15 (1952), 183-88.

8. The characterization of partition lattices J. Sci. Hiroshima Univ. Ser. A., 15 (1952), 189-201.

9. L. R. Wilcox and M. F. Smiley, Metric lattices, Ann. of Math., 40 (1939), 309-27, corrected ibid., 47 (1946), 831.

10. —, A Note on complementation in lattices, Bull. Amer. Math. Soc., 48 (1942), 453-58.

ILLINOIS INSTITUTE OF TECHNOLOGY

UNIVERSITY OF ILLINOIS 



\section{PACIFIC JOURNAL OF MATHEMATICS}

\section{EDITORS}

Ralph S. Phillips

Stanford University

Stanford, California

F. H. BrownelL

University of Washington

Seattle 5 , Washington
A. L. Whiteman

University of Southern California Los Angeles 7. California

L. J. PAIGE

University of California

Los Angeles 24, California

ASSOCIATE EDITORS
E. F. BECKENBACH
D. DERRY
H. L. ROYDEN
E. G. STRAUS
T. M. CHERRY
M. OHTSUKA
E. SPANIER
F. WOLF

\section{SUPPORTING INSTITUTIONS}

UNIVERSITY OF BRITISH COLUMBIA

CALIFORNIA INSTITUTE OF TECHNOLOGY

UNIVERSITY OF CALIFORNIA

MONTANA STATE UNIVERSITY

UNIVERSITY OF NEVADA

NEW MEXICO STATE UNIVERSITY

OREGON STATE COLLEGE

UNIVERSITY OF OREGON

OSAKA UNIVERSITY

UNIVERSITY OF SOUTHERN CALIFORNIA
STANFORD UNIVERSITY

UNIVERSITY OF TOKYO

UNIVERSITY OF UTAH

WASHINGTON STATE COLLEGE,

UNIVERSITY OF WASHINGTON

AMERICAN MATHEMATICAL SOCIETY

CALIFORNIA RESEARCH CORPORATION

HUGHES AIRCRAFT COMPANY

SPACE TECHNOLOGY LABORATORIES

NAVAL ORDNANCE TEST STATION

Printed in Japan by International Academic Printing Co., Ltd., Tokyo, Japan

Reprinted 1966 in the United States of America 


\section{Pacific Journal of Mathematics}

\section{Vol. 11, No. $1 \quad$ November, 1961}

A. A. Albert, Generalized twisted fields ............................ 1

Richard Arens, Operational calculus of linear relations ................... 9

John Herbert Barrett, Disconjugacy of a self-adjoint differential equation of the fourth order ....................................... 25

Paul Richard Beesack, Hardy's inequality and its extensions ............... 39

Julius Rubin Blum and David Lee Hanson, On invariant probability measures.

II .............................................

Robert Allen Bonic, Symmetry in group algebras of discrete groups.......... 73

R. Creighton Buck, Multiplication operators ...................... 95

Jack Gary Ceder, Some generalizations of metric spaces ................. 105

Meyer Dwass, Random crossings of cumulative distribution functions ......... 127

Albert Edrei, Wolfgang H. J. Fuchs and Simon Hellerstein, Radial distribution and

deficiencies of the values of a meromorphic function ............... 135

William Cassidy Fox, Harmonic functions with arbitrary local singularities ..... 153

Theodore Thomas Frankel, Manifolds with positive curvature ............... 165

Avner Friedman, A strong maximum principle for weakly subparabolic

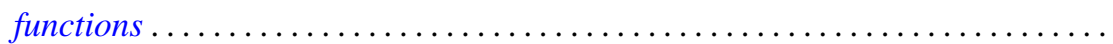

Watson Bryan Fulks and J. O. Sather, Asymptotics. II. Laplace's method for

multiple integrals ......................................

Adriano Mario Garsia and Eugene Richard Rodemich, An embedding of Riemann

surfaces of genus one ..................................... 193

Irving Leonard Glicksberg, Weak compactness and separate continuity......... 205

Branko Grünbaum, On a conjecture of H. Hadwiger .................. 215

Frank J. Hahn, On the action of a locally compact group on $E_{n} \ldots \ldots \ldots \ldots \ldots . . \ldots 221$

Magnus R. Hestenes, Relative hermitian matrices ..................... 225

G. K. Kalisch, On similarity invariants of certain operators in $L_{p} \ldots \ldots \ldots \ldots .247$

Yitzhak Katznelson and Walter Rudin, The Stone-Weierstrass property in Banach

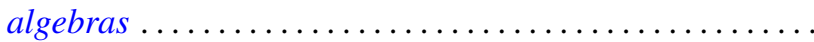

Samir A. Khabbaz, The subgroups of a divisible group $G$ which can be represented as intersections of divisible subgroups of $G \ldots \ldots \ldots \ldots \ldots \ldots \ldots \ldots \ldots . \ldots \ldots 7$

Marvin Isadore Knopp, Construction of a class of modular functions and forms .......................................... 275

Charles Alan McCarthy, Commuting Boolean algebras of projections .......... 295

T. M. MacRobert, Transformations of series of E-functions ................ 309

Heinz Renggli, An inequality for logarithmic capacities ................. 313

M. S. Robertson, Applications of the subordination principle to univalent functions .......................................... 315

David Sachs, Partition and modulated lattices ..................... 325

Frank S. Scalora, Abstract martingale convergence theorems ............... 347

Elbert A. Walker, Torsion endomorphic images of mixed Abelian groups ........ 375

Morgan Ward, The prime divisors of Fibonacci numbers................. 379

Charles R. B. Wright, On the nilpotency class of a group of exponent four....... 387 